Journal of Mathematics and Statistics 2 (3): 427-431, 2006

ISSN 1549-3644

(C) 2006 Science Publications

\title{
Passage to the Differences for the BDF's Methods
}

\author{
Abdelouahab Salmi \\ Department of Mathematics, Badji Mokhtar University, Annaba, Algeria
}

\begin{abstract}
In this study, we give an improvement to the Backward Differentiation Formula (BDF). We use a formula for the differences that permits us to determine the new coefficients $\hat{\alpha}_{i}$ and $\hat{\gamma}_{i}$ in function of the classical coefficients $\alpha_{i}$ and $\gamma_{i}$ of this method. Our proposed approach decreases the error and the cost of computation. Numerical results for concrete problems are given to support the theory.
\end{abstract}

Key words: Stiff systems, backward differentiation formula, differential-algebraic equations

\section{INTRODUCTION}

We are interested in initial value problems for the Differential-Algebraic Equation (DAE) of the form:

$F\left(x, x^{\prime}, t\right)=0$

Where $F, x$ and $x^{\prime}$ are s-dimensional vectors, $F$ is assumed to be differentiable. We investigate some of the practical difficulties involved with implementing the BDF, for the DAE of the form,

$0=F_{1}\left(x, x^{\prime}, y, t\right), 0=F_{2}(x, y, t)$

and the implicit differential equation of the form:

$F\left(x, x^{\prime}, t\right)=0$

Where the initial values of $x$ and $y$ are given, $\frac{\partial F}{\partial x^{\prime}}$ is non-singular. The function $F$ is assumed to be sufficiently smooth. The Jacobian matrix may be singular. As a special case, if $\frac{\partial F}{\partial x^{\prime}}$ is non-singular, equation (2) is locally a system of ODE's. However, if the Jacobian is singular, equation (2) is in fact a system of DAE's. In such a system, there are algebraic constraints on the variables. Systems of this form arise frequently in the modelling of engineering problems, for example the simulation of electrical networks, the solution of certain equations in fluid dynamics and the simulation of mechanical systems ${ }^{[1-3]}$. Other areas of application are: control theory ${ }^{[2]}$, power systems ${ }^{[3]}$ and heat flow ${ }^{[2]}$.

For the stiff problems ${ }^{[4,5]}$ and differential-algebraic equations, our approach are based on BDF's ${ }^{[5,6]}$ :

$$
\begin{aligned}
& \rho x_{n+1}=x_{n+1}^{\prime}=-\frac{1}{h} \sum_{i=0}^{k} \alpha_{i} x_{n+1-i}, k=\overline{1,6} \\
& x_{n+1}^{p}=\sum_{i=1}^{k+1} \gamma_{i} x_{n+1-i}, \quad k=\overline{1,6}
\end{aligned}
$$

Where $h=t_{n+1}-t_{n}$ and the coefficients $\alpha_{i}$ and $\gamma_{i}$ depend on the stepsize $h$ and the order $k$.

This type of methods was studied $\mathrm{in}^{[1,7]}$. The contribution that we bring here to the BDF's permits the decrease of the global error as well as the explicit dependence of the coefficients $\alpha_{i}$ and $\gamma_{i}$ on the stepsize $h$ and the order $k^{[5,6]}$.

The basic idea of using BDF's for solving DAE systems was introduced by $\mathrm{Gear}^{[2]}$ and consists of replacing $x^{\prime}$ in (1) by a difference approximation and then solving the resulting equations for approximations to $x$ and $y$. The simplest example of numerical ODE method for (1) is the backward Euler method. Using this approach, the derivative $x^{\prime}\left(t_{n+1}\right)$ at time $t_{n+1}$ is approximated by a backward difference of $x(t)$ and the resulting system of nonlinear equations for $x_{n+1}$ and $y_{n+1}$ is,

$$
\begin{gathered}
0=F_{1}\left(x_{n+1}, \frac{x_{n+1}-x_{n}}{t_{n+1}-t_{n}}, y_{n+1}, t_{n+1}\right), \\
0=F_{2}\left(x_{n+1}, y_{n+1}, t_{n+1}\right)
\end{gathered}
$$

The above system has a unique solution ${ }^{[2]}$ if the inverse of scaled Jacobian matrix:

$$
h J_{n}=\left(\begin{array}{cc}
\alpha_{0} \frac{\partial F_{1}}{\partial x^{\prime}}+h \frac{\partial F_{1}}{\partial x} & h \frac{\partial F_{1}}{\partial y} \\
h \frac{\partial F_{2}}{\partial x} & h \frac{\partial F_{2}}{\partial y}
\end{array}\right) \text { exists. }
$$

In an earlier $\operatorname{paper}^{[3,7]}$, it is showed that for the systems under considerations, assumptions must be placed on (1). These assumptions are satisfied in many applications. The k-step constant stepsize BDF converges to order of accuracy $O\left(h^{k}\right)$, where the 
initial values are consistent and the functions are sufficiently smooth. Here we are concerned with the practical difficulties such as varying the stepsize and dealing with-ill conditioned matrices which arise in implementing BDF methods for the solution of (1). An important characteristic for understanding both the properties of solutions to DAE's systems and the behaviour of numerical methods for solving these systems is the index of the system, for which a precise definition is given later in the next section. In order to better understand the index and its role in the structure and solution of DAE's systems, we write:

\section{Assumption (1.1):}

1. The index is less than or equal to one, or

2. The index is less than or equal to two

$$
\text { and } \frac{\partial F_{2}}{\partial y} \equiv 0 \text {, or }
$$

3. The index is less than or equal to three and the system has the form (1).

It is well known that the index of DAE's is a measure of the degree of singularity of the system and also widely regarded as an indication of certain difficulties for numerical methods. Thus DAE's can be difficult to solve when they have a higher index, i.e. an index greater than one ${ }^{[1,2]}$. In this case an alternative treatment is the use of index reduction methods ${ }^{[3,7]}$.

Index and mathematical structure for DAE's: Since a DAE involves a mixture of differentiations and integrations, one may hope that applying analytical differentiations to a given system and eliminating as needed, repeatedly if necessary, will yield an explicit ODE system for all the unknowns. This section presents definitions of DAE's in various forms and introduces the notion of the index.

Definition 2.1: A system of initial value differentialalgebraic equation can be written in its general nonlinear implicit or fully implicit form as in (6a), subject to initial conditions $(6 \mathrm{~b})$,

$F\left(x(t), x^{\prime}(t), t\right)=0$

$x\left(t_{0}\right)=x_{0}, x^{\prime}\left(t_{0}\right)=x_{0}^{\prime}$

Where $F \in I R^{n}, x \in I R^{n}$ and $t \in I R, \quad \frac{\partial F}{\partial x}=F_{x}$ is nonsingular and $\frac{\partial F}{\partial x^{\prime}}=F_{x^{\prime}}$ is singular for all $x(t), x^{\prime}(t)$ and $t$.

The existence of algebraic constraints on the variables is expressed by the singularity of the Jacobian $\frac{\partial F}{\partial x^{\prime}}$ matrix. The initial conditions for $x$ and $x^{\prime}$ defined in (6b) must be consistent in order for (6a) to admit solution. It is this last property that separates DAE's from implicit ODE's. It means that there are algebraic constraints on $x(t)$ in addition to the usual differential equations, in the other words, the components of $x(t)$ are not all independent.

As in the case with both analytical and numerical solutions of ODE's, it is important to classify DAE's (and especially nonlinear DAE's) into forms with known properties and /or methods of solution. In particular, not all DAE's can be solved numerically by classical methods such as $\mathrm{BDF}^{[2,3]}$ or implicit Runge Kutta schemes ${ }^{[3]}$. Thus it is desirable to have structural forms which predict when a DAE will be solvable.

Definition 2.2: For general DAE systems (6), the index along a solution $x(t)$ is the minimum number of differentiation of the system which would be required to solve for $\mathrm{x}^{\prime}$ uniquely in terms of $\mathrm{x}$ and t. (i.e. to define an ODE for $\mathrm{x}$ ). Thus, the index is defined in terms of the over determined system:

$$
\begin{aligned}
& F\left(t, x, x^{\prime}\right)=0 \\
& \frac{d F}{d t}\left(t, x, x^{\prime}, x^{\prime \prime}\right)=0 \\
& \cdots \cdots \ldots \ldots . . \\
& \frac{d^{p} F}{d t^{p}}\left(t, x, x^{\prime}, \ldots, x^{(p+1)}\right)=0
\end{aligned}
$$

to be the smallest integer $p$ such that $\mathrm{x}^{\prime}$ in (7) can be solved in terms of $\mathrm{x}$ and $\mathrm{t}$.

Special DAE forms: The general DAE system (6) can include problems which are not well defined in mathematical sense, as well as problems which result in failure by any direct discretisation method (i.e. a method based on discretisation of $x$ and $x^{\prime}$ without first reformulating the equations). Fortunately, most of the higher- index problems encountered in practice can be expressed as a combination of more restrictive structures of ODEs coupled with constraints. In such systems, the algebraic and the differential variables are explicitly identified for higher-index DAE's as well and the algebraic variables may all be eliminated (in principle) using the same number of differentiations. These are called Hessenberg forms of the DAE and are given below.

\section{Hessenberg index-1}

$x^{\prime}=f(t, x, z)$

$0=g(t, x, z)$

Here, the Jacobian matrix $g_{x}$ is assumed to be non-singular for all $t$. This is also often referred to as a semi-explicit index-1 system.

\section{Hessenberg index-2}

$x^{\prime}=f(t, x, z)$

$0=g(t, x)$

Here, the product of Jacobians $g_{x} f_{z}$ is non-singular for all $t$. This is a pure index-2 DAE. 


\section{Hessenberg index-3}

$x^{\prime}=f(t, x, y, z)$

$z^{\prime}=g(t, x, z)$

$0=h(t, y)$

Here the product of three matrix functions $h_{y} g_{x} f_{z}$ is non-singular for all $t$.

The index has proven to be a useful concept for classifying DAEs in order to construct and identify appropriate numerical methods.

BDF methods: Numerical approache for the solution of differential-algebraic equations can be divided roughly into two classes:

i. Direct discretisations of the given system and (ii) methods which involve a reformulation (e.g. index reduction), combined with discretisation. The desire for as direct a discretisation as possible arises because a reformulation may be costly. It may require more input from the user and it may involve more user intervention. The reason for the popularity of reformulation approach is that, as it turn out, direct discretisations are essentially limited in their utility to index-1 and semi-explicit index-2 DAE systems.

Some numerical methods have been developed, using both $\mathrm{BDF}^{[2,7]}$ and implicit Runge kutta methods ${ }^{[3]}$. These methods are only directly suitable for low index problems and often require that the problem has a special structure. Although many important applications can be solved by these methods, there is a need for more general approach as proposed $\mathrm{in}^{[2,3]}$.

In this paper, our approach is based on the methods of backward differentiation formulas (BDFs):

$$
\begin{aligned}
& \rho x_{n+1}=x_{n+1}^{\prime}=-\frac{1}{h} \sum_{i=0}^{k} \alpha_{i} x_{n+1-i}, k=\overline{1,6} \\
& x_{n+1}^{p}=\sum_{i=1}^{k+1} \gamma_{i} x_{n+1-i}, \quad k=\overline{1,6}
\end{aligned}
$$

This type of methods has been studied by ${ }^{[1,7]}$; the contribution that we bring to the BDF's, permits the decrease of the global error as well as the explicit dependence of the coefficients $\alpha_{i}$ and $\gamma_{i}$ on the step $h$ and the order $k^{[5,6]}$.

Computation of the coefficients $\alpha_{i}$ and $\gamma_{i}$ : The coefficients $\alpha_{i}$ depend on the values $t_{n-1}, t_{n}, \ldots, t_{n+1-k}$ and are determined so that the formula (1) gives an exact result for $x(t)=p_{i}(t)=\left(\frac{t_{n+1}-t}{h}\right)^{i}[4,6]$.

While substituting the polynomials $p_{i}(t)$ in (8) and (9), one gets the following linear system:

$$
V_{1} \alpha=e_{2} \text { and } V_{2} \gamma=e_{1}
$$

With $e_{1}=(1,0, \ldots, 0)^{T}, e_{2}=(0,1, \ldots, 0)^{T}$, $\alpha=\left(\alpha_{0}, \alpha_{1}, \ldots, \alpha_{k}\right)^{T}$ and $\gamma=\left(\gamma_{1}, \gamma_{1}, \ldots, \gamma_{k+1}\right)^{T}$.

The matrices $V_{1}$ and $V_{2}$ are of Vendermonde type:

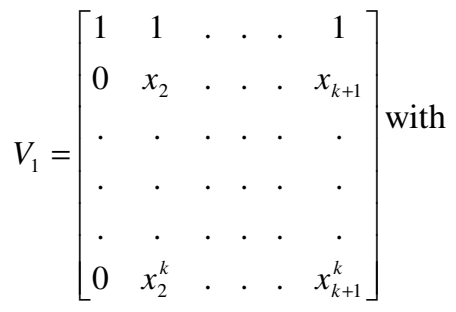

$x_{i}=\frac{t_{n+1}-t_{n+2-i}}{h}, i=\overline{1, k+1}$

And

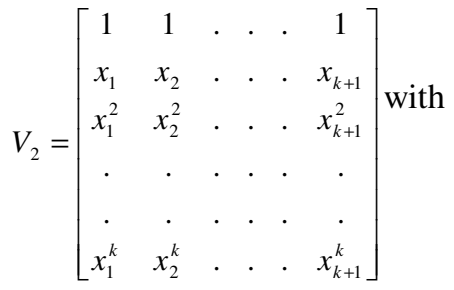

$$
\begin{aligned}
& x_{i}=\frac{t_{n+1}-t_{n+1-i}}{h}, i=\overline{1, k+1} \text {. }
\end{aligned}
$$

While taking into account the specific pace of these systems, we can find the coefficients with a reduced computation $\operatorname{cost}^{[5,6]}$.

Proposition 3.1 ${ }^{[6]}$ : The coefficients $\gamma_{i}$ and $\alpha_{i}$ are given by,

$\gamma_{i}=\prod_{i \neq \nu=1}^{k+1} \frac{t_{n+1}-t_{n+1-\nu}}{t_{n+1-i}-t_{n+1-\nu}} \quad, i=\overline{1, k+1}$

$\alpha_{0}=-\sum_{v=1}^{k} \frac{t_{n+1}-t_{n}}{t_{n+1-i}-t_{n+1-v}}$

and

$\alpha_{i}=\frac{t_{n+1}-t_{n}}{t_{n+1}-t_{n+1-i}} \prod_{i \neq v=1}^{k} \frac{t_{n+1}-t_{n+1-\nu}}{t_{n+1-i}-t_{n+1-\nu}} \quad, i=\overline{1, k}$

The formulas (11) and (12) can be used for the computation of the coefficients for different steps and an $\operatorname{order} 1 \leq k \leq 6^{[5]}$.

Passage to the differences: To decrease the values of rounded errors when using the formulas (8) and (9), we replace the values $x_{n+1-i}$ in (8) and (9) by the differences: $\Delta x_{n-i}=x_{n+1-i}-x_{n-i}$. In this case the formulas (8) and (9) take the following form:

$$
\begin{aligned}
& x_{n+1}^{\prime}=-\frac{1}{h} \sum_{i=0}^{k-1} \hat{\alpha}_{i} \Delta x_{n-i}, k=\overline{1,6} \\
& x_{n+1}^{p}=x_{n}+\sum_{i=1}^{k} \hat{\gamma}_{i} \Delta x_{n-i}, k=\overline{1,6}
\end{aligned}
$$


Computation of the coefficients $\hat{\alpha}_{i}$ and $\hat{\gamma}_{i}$

Proposition 3.2: The coefficients $\hat{\alpha}_{i}$ and $\hat{\gamma}_{i}$ are determined from the coefficients $\alpha_{i}$ and $\gamma_{i}$ as follows:

$\hat{\alpha}_{0}=\alpha_{0}$ and $\hat{\alpha}_{i}=\sum_{j=0}^{i} \alpha_{j}, i=\overline{1, k-1}$

$\hat{\gamma}_{1}=\gamma_{1}-1$ and $\hat{\gamma}_{i}=\sum_{l=1}^{i} \gamma_{l}, i=\overline{2, k}$

Proof: According to formulas (8) and (13), we get:

$\sum_{i=0}^{k} \alpha_{i} x_{n+1-i}=\sum_{i=0}^{k-1} \hat{\alpha}_{i} x_{n+1-i}-\sum_{i=0}^{k-1} \hat{\alpha}_{i} x_{n-i} \quad, k=\overline{1,6}$

By identification, we get:

$$
\begin{aligned}
& \hat{\alpha}_{0}=\alpha_{0} \\
& \hat{\alpha}_{1}-\hat{\alpha}_{0}=\alpha_{1} \\
& \cdot \\
& \hat{\alpha}_{k-1}-\hat{\alpha}_{k-2}=\alpha_{k-1} \\
& \quad-\hat{\alpha}_{k-1}=\alpha_{k}
\end{aligned}
$$

And the general formula of the coefficients $\hat{\alpha}_{i}$ is:

$\hat{\alpha}_{j}=\sum_{i=0}^{j} \alpha_{i}, j=\overline{0, k-1}$.

In the same way we get the coefficients $\hat{\gamma}_{i}$, from the equality:

$\sum_{i=1}^{k+1} \gamma_{i} x_{n+1-i}=x_{n}+\sum_{i=1}^{k} \hat{\gamma}_{i} \Delta x_{n-i}$

We get the formula:

$\hat{\gamma}_{j}=-1+\sum_{i=1}^{j} \gamma_{i}, j=\overline{1, k}$.

Remark 3.1: While taking account of $\sum_{i=1}^{k+1} \gamma_{i}=0$ and $\sum_{i=0}^{k} \alpha_{i}=0^{[5]}$, we find that:

$\hat{\alpha}_{j}=-\sum_{i=j+1}^{k} \alpha_{i}, j=\overline{0, k-1}$ and

$\hat{\gamma}_{j}=-\sum_{i=j+1}^{k} \gamma_{i}, j=\overline{1, k}$

Modification of the value of the step and the order: We are going to show the algorithm of the choice of the step and the order for the backward differentiation method.

Let us designate by $E_{\max }$ the error of tolerance on one interval of integration of length $\mathrm{L}$ and by $e\left(h_{n+1}\right)$ the error of discretisation for the step $h_{n+1}$.

Then

$e\left(h_{n+1}\right)=E_{\max } \frac{h_{n+1}}{L}$
One supposes that the derivative of order k, $x^{(k+1)}$ remain unaltered for two successive steps, the truncation error for the step $h_{n+1}$ is: $\varepsilon_{k}\left(h_{n+1}\right) \cong \eta_{k}^{k+1} \varepsilon_{k}\left(h_{n}\right)$, with $\eta_{k}=\frac{h_{n+1}}{h_{n}}$.

The new step and the order $k$ are chosen such that $h$ is maximal and verifying: $\varepsilon_{k} / h \leq E_{\max } / L^{[2,3]}$. The peak value of the step $h_{n+1}$ can be found from the relation: $\varepsilon_{k}\left(h_{n+1}\right)=e\left(h_{n+1}\right)$, where

$\eta_{k}=\sqrt[k]{\frac{E_{\max } h_{n}}{\left|\varepsilon_{k}\left(h_{n}\right)\right| L}}$

In practice $\eta_{k}$ is computed for the order $k^{\prime}=k-1, k$ and $k+1$ and in continuation, one chooses the method that permits to make the maximal step $h_{n+1}$ : $h_{n+1}=\eta h_{n}$ with $\eta=\max \left(\eta_{k-1}, \eta_{k}, \eta_{k+1}\right)$.

The formula (18) makes forecast of the value of the error of discretisation only for the step $h_{n+1}$. Actually, it is a lot bigger than the local truncation error; it is the reason for which the proposed algorithm plans the dismissal of steps.

\section{RESULTS}

There exist convergence results, In particular; the $k$-step BDF method of fixed step size $h$ for $k<7$; converges to $O\left(h^{k}\right)$ if all initial values are correct to $O\left(h^{k}\right)$ and if the Newton iteration on each step is solved to accuracy $O\left(h^{k+1}\right)^{[1]}$. This convergence result has also been extended to variable step size BDF methods, provided that they are implemented in such a way that the method is stable for standard Odes. This convergence result extends to semi-explicit index-2 $\operatorname{DAEs}^{[7]}$.

In this section we carry some numerical tests on simple, but interesting problems. In example 4.1, we use " $E_{\max }^{x} "$, to denote the maximum over all components of the error in $x$ and " $E_{\max }^{y}$ " denotes the maximum of the error in $y$. This example is solved, directly using BDF with the formulas (11), (12) and using the BDF with the formulas (15), (16).

Example 4.1 (Differential-algebraic equation): Consider for $0 \leq \mathrm{t} \leq 1$, the system:

$\left\{\begin{array}{l}x_{1}^{\prime}=(2-t) v y+q_{1}(t) \\ x_{1}^{\prime}=(v-1) y+q_{2}(t) \\ 0=(t+2) x_{1}+\left(t^{2}+4\right) x_{2}+r(t)\end{array}\right.$ 
Table 1: Errors in $\mathrm{x}$ and $\mathrm{y}$ for example $4.1 \mathrm{using}$ BDF with a difference.

\begin{tabular}{lccc}
\hline BDF & \multicolumn{1}{c}{ BDF with difference } \\
$E_{\max }^{x}$ & $E_{\max }^{y}$ & $E_{\max }^{x}$ & $E_{\max }^{y}$ \\
\hline $5.0000 \mathrm{E}-07$ & $7.1000 \mathrm{E}-07$ & $4.8990 \mathrm{E}-08$ & $7.0000 \mathrm{E}-07$ \\
$3.9000 \mathrm{E}-11$ & $4.2000 \mathrm{E}-11$ & $3.8700 \mathrm{E}-12$ & $4.1100 \mathrm{E}-12$ \\
$1.8000 \mathrm{E}-15$ & $1.8000 \mathrm{E}-16$ & $1.8200 \mathrm{E}-16$ & $1.8200 \mathrm{E}-16$ \\
$4.6100 \mathrm{E}-15$ & $6.4000 \mathrm{E}-17$ & $4.5900 \mathrm{E}-17$ & $6.3700 \mathrm{E}-17$ \\
\hline
\end{tabular}

Table 2: Convergence of the BDF scheme with a difference for Stiff problem.

\begin{tabular}{|c|c|c|c|c|}
\hline \multirow{3}{*}{$\begin{array}{l}\text { Tolerance } \\
\text { Method } \\
\text { Values/Errors }\end{array}$} & \multicolumn{3}{|c|}{$\varepsilon=10^{-3}$} & \multirow[b]{3}{*}{$E_{2}$} \\
\hline & BDF5 & & BDF6 & \\
\hline & $\mathrm{E}_{1}$ & $\mathrm{E}_{2}$ & $\mathrm{E}_{1}$ & \\
\hline $\mathrm{t}=2.0$ & 7.5951 E-03 & $2.0934 \mathrm{E}-03$ & 5.3060 E-03 & 2.4746 E-05 \\
\hline$t=3.0$ & 6.4092 E-03 & $7.1329 \mathrm{E}-04$ & $4.2336 \mathrm{E}-03$ & 7.8208 E-04 \\
\hline$t=6.0$ & $6.6100 \mathrm{E}-03$ & 2.7391 E-04 & 5.0564 E-03 & 2.1270 E-03 \\
\hline $\begin{array}{l}\text { Tolerance } \\
\text { Method } \\
\text { Values/Errors }\end{array}$ & BDF5 & $\varepsilon=10^{-4}$ & BDF6 & \\
\hline $\mathrm{t}=2.0$ & $1.4042 \mathrm{E}-03$ & $3.5832 \mathrm{E}-04$ & $3.5691 \mathrm{E}-04$ & $2.0970 \mathrm{E}-04$ \\
\hline$t=3.0$ & $1.1559 \mathrm{E}-03$ & $3.1592 \mathrm{E}-04$ & $1.7552 \mathrm{E}-04$ & $6.7362 \mathrm{E}-04$ \\
\hline$t=6.0$ & 3.4594 E-03 & $1.5085 \mathrm{E}-05$ & $2.9635 \mathrm{E}-03$ & 2.8997 E-04 \\
\hline
\end{tabular}

with $x_{1}(0)=1 \quad$ and $q(t)=\left(\begin{array}{l}(v+1) e^{t} \\ (1+(v-1) /(2-t)) e^{t}\end{array}\right)$,

$r(t)=-\left(t^{2}+t-2\right) e^{t}$.

The exact solution is: $x_{1}=x_{2}=e^{t}, y=-e^{t} /(2-t)$; the numerical results with $v=1000$ is given in Table 1 .

The comparison between the general results given in table 1 and the published results in ${ }^{[7]}$ and $^{[6]}$ shows the advantage of using the proposed methods of this paper, for this example.

Example 4.2 (Stiff problem):

$\left\{\begin{array}{l}x_{1}^{\prime}=-30 x_{1}+29 x_{2}+3 \\ x_{2}^{\prime}=70 x_{1}-70 x_{2}, t \in[0,10] \\ \text { avec } x_{1}(0)=x_{2}(0)=1\end{array}\right.$

In Table 2, we give the maximum over all components of the errors $\left(E_{1}\right)$ and the troncature error $\left(E_{2}\right)$ evaluated at $\mathrm{t}=2.0, \mathrm{t}=3.0$ and $\mathrm{t}=6.0$. This example is solved, using BDF with the formulas (15) and (16).

For a tolerance $\varepsilon \leq 10^{-3}$, BDF5 and BDF6 converge, but BDF6 has a faster convergence rate than BDF5. The comparison between the general results mentioned in Table 2 and the published results in ${ }^{[6]}$ shows the advantage of using the proposed methods of this paper, for this example.

\section{CONCLUSION}

The implementation of the numerical methods analyzed in this article shows that the results found by our approach of BDF's, as using the formula to the differences, is better than those of ${ }^{[4]}$ and $^{[6]}$; and can be improved more in maintaining the same order for the following steps and while using a good approximation of the Jacobian matrix ${ }^{[7]}$. This improvement can overcome the problem of instability that can be met for small tolerances.

\section{ACKNOWLEDGEMENT}

This work was supported in part by the accord program CMEP number 04 MDU 606.

\section{REFERENCES}

1. Brenan, K.E. and B.E. Enquist, 1988. Backward differentiation approximation of nonlinear differential algebraic equations and supplement S7S17. Math. Comp., 51: 659-676.

2. Brenan, K.E., S.L. Campbell and L.R. Petzold, 1995. Numerical Solution Of Initial-Value Problems in Differential-Algebraic Equations. 2nd Edn., SIAM, Philadelphia, PA.

3. Hairer, E. and G. Wanner, 1991. Solving Ordinary Differential Equations II: Stiff and DifferentialAlgebraic Problems. Springer-Verlag.

4. Enright, W.H., T.E. Hull and B. Lendberg, 1975. Comparing numerical methods for stiff systems of Ode's. BIT 15: 10-48.

5. Salmi, A., 1995. Study of some numerical methods for the stiff problems. Magister Thesis. University of Annaba.

6. Nafaa K. and A. Salmi, 1996. Contributions aux méthodes de différentiation en arrière pour les problèmes rigides. Maghreb Math. Rev., 5: 129-149.

7. Lotstedt, P. and L.R. Petzold, 1986. Numerical solution of nonlinear differential equations with algebraic constraints I: Convergence results for backward differential formulas. Math. Comp., 46: 491-516. 